\title{
Editorial
}

\section{Biocompatibility and Toxicity of Nanobiomaterials 2013}

\author{
Xiaoming Li, ${ }^{1}$ Sang Cheon Lee, ${ }^{2}$ Shuming Zhang, ${ }^{3}$ and Tsukasa Akasaka ${ }^{4}$ \\ ${ }^{1}$ Key Laboratory for Biomechanics and Mechanobiology of Ministry of Education, School of Biological Science and Medical Engineering, \\ Beihang University, Beijing 100191, China \\ ${ }^{2}$ Department of Maxillofacial Biomedical Engineering, School of Dentistry, Kyung Hee University, Seoul 130-701, Republic of Korea \\ ${ }^{3}$ Department of Materials Science and Engineering, Johns Hopkins University, Baltimore, MD 21218, USA \\ ${ }^{4}$ Department of Biomedical Materials and Engineering, Hokkaido University, Sapporo 060-8586, Japan
}

Correspondence should be addressed to Xiaoming Li; x.m.li@hotmail.com

Received 16 December 2013; Accepted 16 December 2013; Published 23 January 2014

Copyright (C) 2014 Xiaoming Li et al. This is an open access article distributed under the Creative Commons Attribution License, which permits unrestricted use, distribution, and reproduction in any medium, provided the original work is properly cited.

Up to now, nanomaterials have attracted extensive attention of the researchers in biomedical field [1-3]. Over the past few years, nanobiomaterials have occupied significant position in medical industry. The development of nanobiomaterials for biomedical applications including drug delivery systems, imaging systems for diagnosis, tissue engineering, and dental/bone implant is currently undergoing a dramatic expansion. For successful clinical applications, emerging nanobiomaterials require stringent evaluations for their biological security.

The unique physical and chemical properties, which are seen as special advantages of nanobiomaterials, may cause potentially deleterious effects on human health. According to the different preparation methods, nanobiomaterials can have different size, chemical composition, and shape and can be with or without surface coatings. Each of these factors can affect the interactions between the nanomaterials and cells or tissues.

For example, some nanobiomaterials have been found to be able to concentrate more proteins including specific proteins and these proteins might not only improve cell attachment and proliferation but also differentiate the inducible cells, which promote the tissue repair. Therefore, these nanobiomaterials might have the ability to modulate downstream stem cellular response without exogenous growth factors, coatings, or complex ligand incorporation and have the potential to greatly facilitate the development of tissue engineering and cellular therapies [4], "Biocompatibility and toxicity of nanoparticles and nanotubes" and "The use of nanoscaled fibers or tubes to improve biocompatibility and bioactivity of biomedical materials," [5-7]. However, due to their small size or specific functionalization, nanobiomaterials may cross important biological barriers and cause toxicity to very sensitive systems such as the brain or the developing fetus. Nanobiomaterials designed for drug delivery deliberately overcome biological barriers or they may cross them unintentionally as a result of environmental exposure. Nanobiomaterials can permeate membrane cells and spread along the nerve cells synapses, blood vessels, and lymphatic vascular and they can potentially be deposited in any region of the body, such as liver, spleen, kidney, placenta, the cardiovascular system, and central nervous system (CNS) where they may cause adverse effects [8-10]. That is to say, nanobiomaterials of strong permeability not only provide the effectiveness for the use of them but also give rise to potential threats on the health of human body. Therefore, it is imperative to carry out comprehensive investigations and intensive studies into biocompatibility and toxicity of nanobiomaterials.

Currently, correlation studies on testing biocompatibility and toxicity of nanobiomaterials mainly focus on three levels. At the basic level, tests are performed in vitro in cell or tissue cultures. If the experiment effect is fine, further testing will be conducted next in vivo on animal models before clinical studies are carried out on humans. Existing regulatory frameworks have covered all basic aspects; however, the varying behaviors of nanobiomaterials from the corresponding bulk material strongly suggest the need for adaptation of existing 
frames or specific policies for nanoproducts. This is part of reform for healthy future, and it is imperative to establish risk assessment standards and stipulate safe doses of nanobiomaterials. Unfortunately, now available validated analytical and biological methods as well as certified reference standards for exposure and hazard assessment are far from being enough. Research in future needs to aim at developing certified reference standards, using well-characterized and defined nanobiomaterials, as quality controls additionally to standardized test protocols. Furthermore, a comprehensive explanation of underlying mechanisms on the biocompatibility and toxicity of nanobiomaterials, for example, oxidative stress, immunotoxicity, and genotoxicity, is also needed and possible adverse effects should be considered. In fact, many trials have already been carried out to detect the biocompatibility and toxicity of nanobiomaterials in vitro and in vivo. Based on such preclinical evaluation of biocompatibility and toxicity, current research trends are mainly focused on the distribution of nanobiomaterials in human body and whether the nanobiomaterials induce toxic effects.

In this special issue, dozens of articles are dedicated to the effect of fundamental performance parameter of nanobiomaterials on their biocompatibility and toxicity, the interactions between nanobiomaterials and cell/tissue, immunogenicity and capability of committed differentiation of nanobiomaterials, design of novel biocompatible and biodegradable nanobiomaterials, deep analysis of toxicity mechanism of nanobiomaterials using special instrumentation and technology, and specific challenges to overcome the toxicity of nanobiomaterials. Not only through accurate data and updated reviews on the above aspects could readers get access to in this special issue, but also the chance to get a clear idea of how the properties such as dimensions, size, shape, chemical composition, surface chemistry, and coatings affect the biocompatibility and toxicity of nanobiomaterials, the mechanisms of interaction between nanobiomaterials and organs, tissues, and cells, and the self-assembly mechanism of natural tissues in order to better biomimetically prepare nanobiomaterials for tissue engineering, to better functionalize nanobiomaterials using chemical method or to enhance the biological activity of nanobiomaterials by introducing various molecules, growth factors, antigens, and specific DNA sequences, and most importantly to establish standard sets of toxicological tests for nanobiomaterials that can be used as an effective way to estimate the safety of nanobiomaterials.

\section{Xiaoming Li Sang Cheon Lee Shuming Zhang Tsukasa Akasaka}

[3] X. Li, H. Gao, M. Uo et al., "Maturation of osteoblast-like SaoS2 induced by carbon nanotubes," Biomedical Materials, vol. 4, no. 1, Article ID 015005, 2009.

[4] X. Li, L. Wang, Y. Fan, Q. Feng, F. Cui, and F. Watari, "Nanostructured scaffolds for bone tissue engineering," Journal of Biomedical Materials Research A, vol. 101, no. 8, pp. 24242435, 2013.

[5] X. Li, C. A. van Blitterswijk, Q. Feng, F. Cui, and F. Watari, “The effect of calcium phosphate microstructure on bone-related cells in vitro," Biomaterials, vol. 29, no. 23, pp. 3306-3316, 2008.

[6] X. Li, H. Liu, X. Niu et al., "The use of carbon nanotubes to induce osteogenic differentiation of human adipose-derived MSCs in vitro and ectopic bone formation in vivo," Biomaterials, vol. 33, no. 19, pp. 4818-4827, 2012.

[7] X. Li, H. Liu, X. Niu et al., "Osteogenic differentiation of human adipose-derived stem cells induced by osteoinductive calcium phosphate ceramics," Journal of Biomedical Materials Research $B$, vol. 97, no. 1, pp. 10-19, 2011.

[8] X. Li, X. Liu, J. Huang, Y. Fan, and F.-Z. Cui, "Biomedical investigation of CNT based coatings," Surface and Coatings Technology, vol. 206, no. 4, pp. 759-766, 2011.

[9] X. Li, Y. Fan, and F. Watari, "Current investigations into carbon nanotubes for biomedical application," Biomedical Materials, vol. 5, no. 2, Article ID 022001, 2010.

[10] M. Dusinska, Z. Magdolenova, and L. Fjellsbo, "Toxicological aspects for nanomaterial in humans," Methods in Molecular Biology, vol. 948, pp. 1-12, 2013.

\section{References}

[1] X. Li, Q. Feng, X. Liu, W. Dong, and F. Cui, “Collagen-based implants reinforced by chitin fibres in a goat shank bone defect model," Biomaterials, vol. 27, no. 9, pp. 1917-1923, 2006.

[2] X. Li, H. Gao, M. Uo et al., "Effect of carbon nanotubes on cellular functions in vitro," Journal of Biomedical Materials Research A, vol. 91, no. 1, pp. 132-139, 2009. 

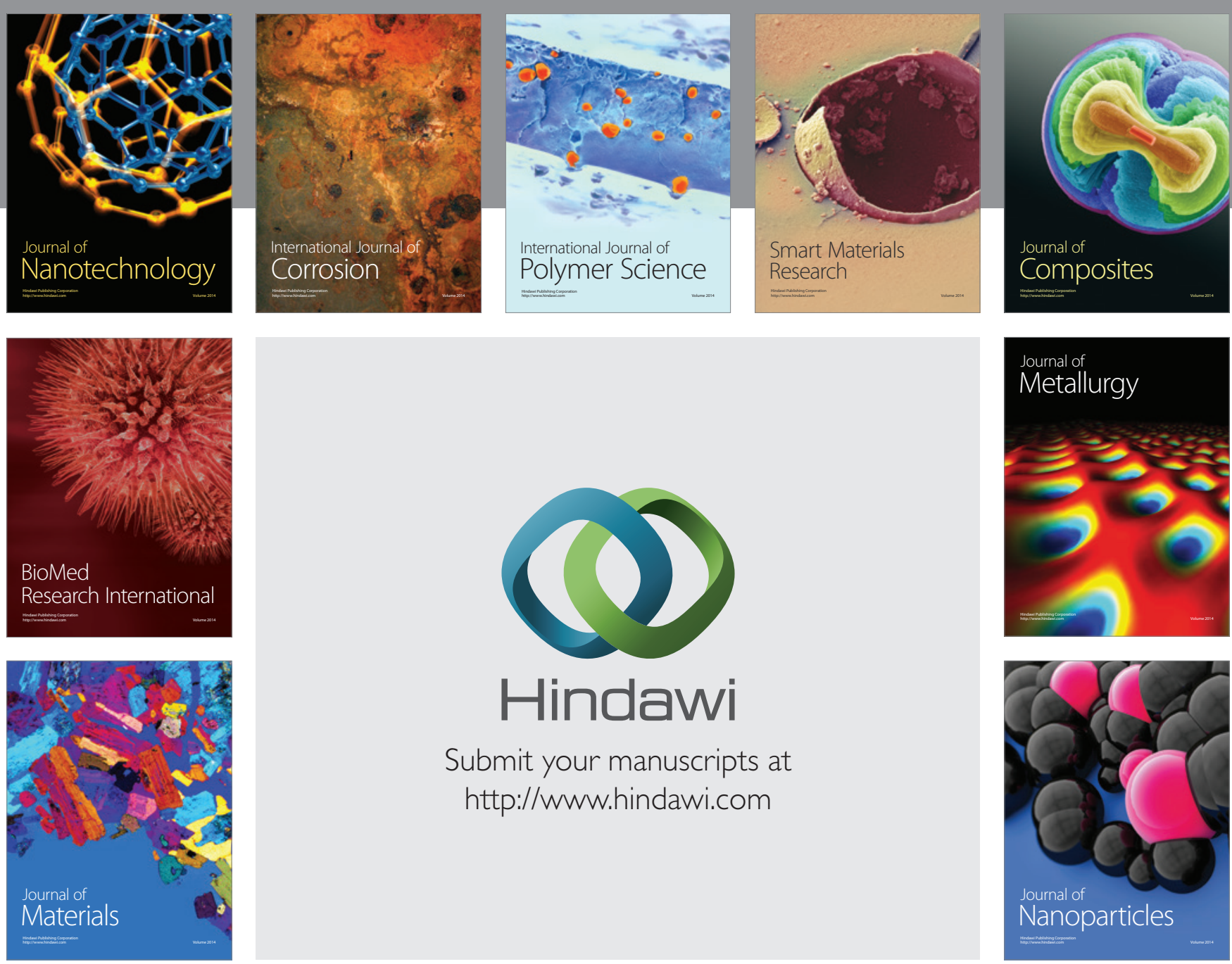

Submit your manuscripts at http://www.hindawi.com
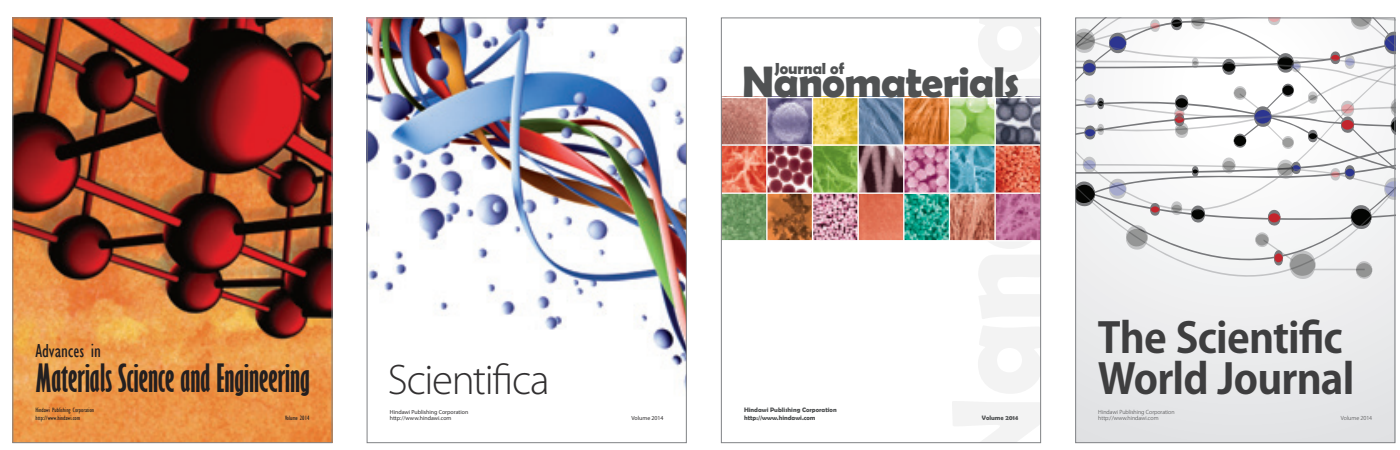

\section{The Scientific World Journal}
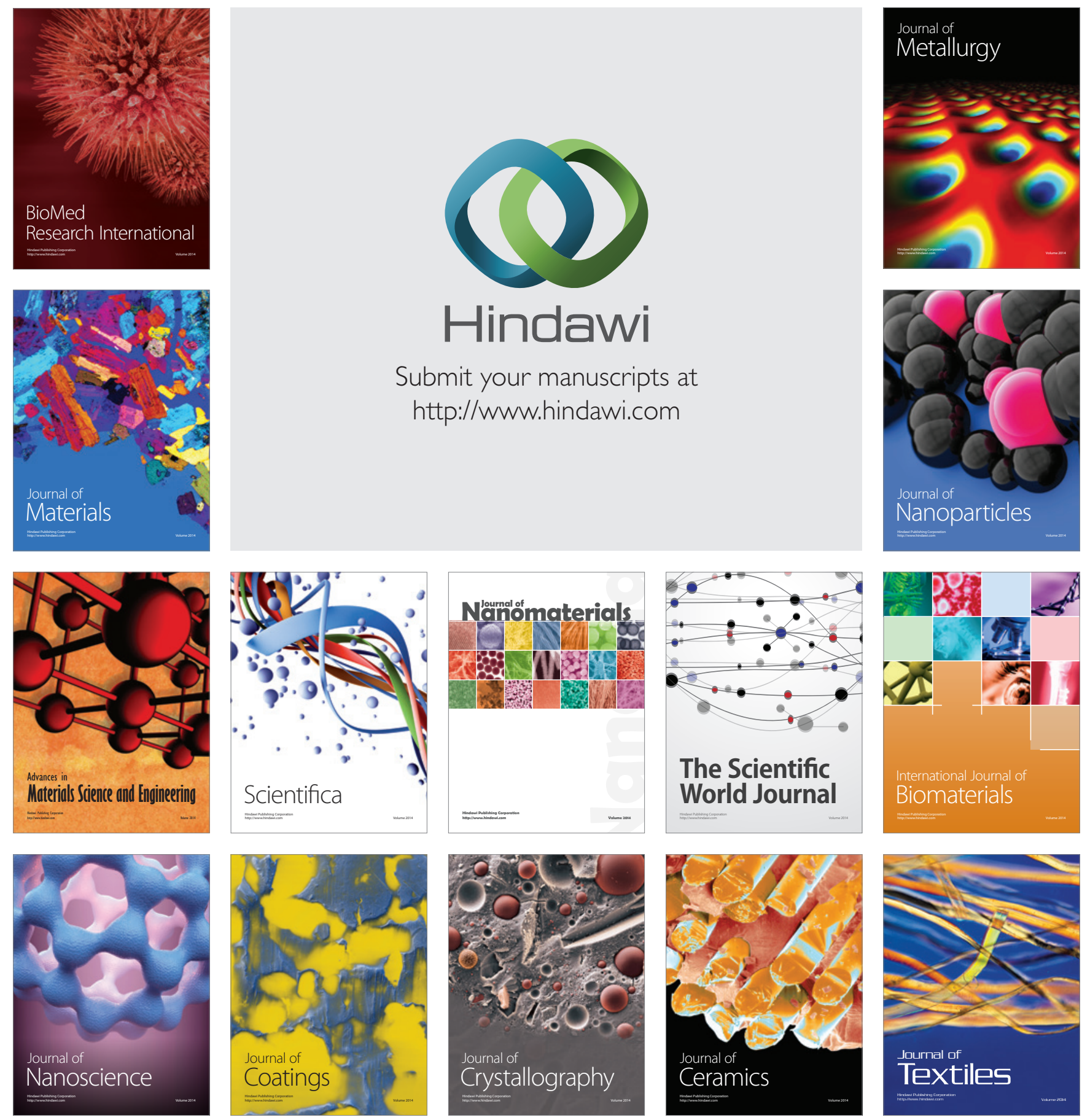